\title{
Theoretical basics of process of the dispersal system correction
}

\author{
Natalia Khrunina $^{1 *}$ and Svetlana Korneeva ${ }^{1}$ \\ ${ }^{1}$ Mining Institute FEB RAS, 51 Turgenev st., Khabarovsk, 680000, Russia
}

\begin{abstract}
Annotation. Factors analysis, determining the stability of mineral dispersal systems during disintegration was done. A mathematical model of the process of formation of small particles of the mineral component of hydromineral mixture in the field of hydrodynamic influence is presented, initiated by cavitation. The identified basic dependency options allowed, experimental studies of the disintegration of mineral particles, using electron microscopy to control the process and assess its effectiveness. Developed ideas about structural and mechanical features of mineral hydro-mineral mixture taking into account thermodynamic potential, the preconditions are not only theoretical justification for the process, but also the development of applied research in the development of technologies and means of processing and enrichment minerals in environmentally friendly ways.
\end{abstract}

Keywords: hydrodynamic impact, clay particles, hydro-mineral mixture, cavitation, ultrasound, thermodynamic potential

\section{Introduction}

Currently, development of gold-bearing polyminereal deposits In the Far East hampered by the low productivity of the mineral extraction and processing process, and - completeness of extraction of small fractions of valuable components. Deposits are involved, most of which can be attributed by the content of valuable components to hard-to-concentration gravitational methods due to the significant content of small and thin fractions. At the same time, the high content in clay deposits determines the search for new methods of influence and determines the study of the patterns of their influence.

Polyminerality of sands scatterings, high clay, pollution of the natural environment and increasing demands to increase the completeness of extraction of minerals from the bowels determines the relevance of the solution to the problem of mining processing, taking into account all these aspects with the first of all a disintegrating effect to extract small and fine particles less than $0.005 \mathrm{~mm}$ in size. The development of effective and safe processes can play a significant role in solving this problem, based on new physical principles of impact on sands and their hydro-mineral mixture [1-6].

An important aspect of the research is the analysis of various physical phenomena and effects, occurring with a variety of physical effects on mineral components to determine the prospect of practical application, and to gain new knowledge or to determine the prospect of new information and the discovery of new phenomena.

\footnotetext{
*Corresponding authors: npetx@mail.ru
} 


\section{Theoretical aspects of the sustainability of dispersal systems}

The efficiency of the process of extraction of valuable components is conditioned by the ability to evaluate and manage the process of directional change of properties of sand-clay rocks and physics - chemical parameters of recyclable sands in the process of disintegration. It is known that when particles converge in the aquatic environment under the influence of hydrodynamic influence, their immediate clumping is hindered by the forces of repulsion. One of the most factors determining the stability of dispersed systems is the electrostatic interaction of diffuse layers. Because outside the double electric layer (DEL) the position of the electrostatic field of particles is zero, the repulsion forces appear when the diffuse layers of particles are blocked [1]. Changes in cations concentration and particle potential within this layer affect the stability of dispersed particles. The main provisions of this theory are based on the works G. Gui, J. Chapman, O. Stern, A.N. Frumkin, D. Graham, etc. Based on Boltzmann's equation, a change in the concentration of ions within the DEL was calculated, and dependence was obtained [1]

$$
n_{i}=n_{i_{0}} \exp \left(-\frac{\mathrm{Z} e \varphi}{k T}\right)
$$

where $n_{i}$ - concentration of ions; $n_{i_{0}}$ - concentration of ions outside the diffusion layer; $Z$ Ion valence; $e$ - single electrostatic charge $\left(16 \cdot 10^{-20} \mathrm{C}\right) ; \varphi$ - electrical potential at the point; $k$ - Boltzmann's constant $\left(1,38 \cdot 10^{-23} \mathrm{~J} /{ }^{0} \mathrm{~K}\right) ; T$ - temperature, ${ }^{0} \mathrm{~K}$.

From the equation (1) it follows that, as you move away from the surface, the concentration of ions, the charge of which is the opposite to the charge of the surface, decreases, and the concentration of ions charged of the same name with the surface (signs $Z$ and $\varphi$ same), downgraded and increasing. The change in potential in a flat surface is described by the Poisson-Boltzmann equation [1]

$$
\frac{d^{2} \varphi}{d x^{2}}=-\frac{4 \pi}{\varepsilon} \sum \mathrm{Zen}_{i_{0}} \exp \left[-\frac{\mathrm{Z} e \varphi}{k T}\right],
$$

where $x$ - distance from particle surface; $\varepsilon$ - dielectric permeability of the environment.

Once the equation is simplification, it is

$$
\frac{d^{2} \varphi}{d x^{2}}=-\frac{2 \mathrm{Z} e n_{i_{0}}}{\varepsilon} s h\left[-\frac{\mathrm{Z} e \varphi}{k T}(x)\right]
$$

The possibility of applying this equation to clay systems was considered by G. Bolt and H. Van Olfen. Since the theory was based on the concept of permanent surface potential, it can be applied to clay systems for side-prick particles [1].

For flat-shaped clay particles larger than the diffusion layer, stability is determined by function

$$
\varphi(x) \approx \frac{4 k T}{\mathrm{Z} e} \operatorname{th}\left(\frac{\mathrm{Z} e \varphi_{0}}{4 k T}\right) e^{-\chi x},
$$

where $\chi$ - diffuse layer parameter, related to other characteristics, the ratio

$$
\frac{1}{\chi}=\sqrt{\frac{\varepsilon k T}{8 \pi \mathrm{Z}^{2} e^{2} n_{0}}} .
$$

In here $\varepsilon$ - dielectric permanent environment; $T$ - temperature; Z- Ion valence; $n_{o}$ - electrolyte concentration in solution; $\varphi_{o}$ - electrical potential in the gap between particles. 


\section{Theoretical justification for the formation of small particles of mineral component in the wave impact initiated by cavitation}

The Gibbs equation was adopted as a baseline equation to theoretically substantiate the formation of small particles in the wave. According to this equation, the change in thermodynamic potential $d E$ systems are presented as [2]

$$
d E=-\Omega \cdot d T+V d p+\sigma d S+\sum \mu d N+F \sum z \varphi d N,
$$

where is $\Omega$-entropy particles; $T$ - particle temperature; $V$ - particle volume; $p$ - pressure inside particles; $\sigma-$ specific surface energy of particles; $S$ - area of the interphase surface of particles; $\mu$ - the chemical potential of the surface components of the system when particles interact with each other in the presence of water; $N$ - number of moles of all surface particle components; $F$ - constant Faraday, $F=9,648456 \cdot 10^{4} \mathrm{C} / \mathrm{mol} ; Z$ - charge of particles of surfaces of a certain grade; $\varphi$ - the electrical potential of the surface part of the system containing particles of a certain type, or the internal potential of different phases by chemical composition.

In the environment under consideration, there is a change in the aggregate state of substances that are predominantly condensed (solid and liquid) and a small amount in the gaseous, so the differences between changes in the internal energy of particles and changes in entropy $\Omega$ of systems of these particles are insignificant. Given the assumptions taken, the equation can be given the appearance of

$$
d E-\sum \mu d N-F \sum z \varphi d N=\sigma d S
$$

Physical-mechanical and structural changes increase as the duration of grinding $t$ increases and is determined by the amount of energy being reduced

$$
d E=W \cdot \eta d t
$$

where $W$-power emitted energy; $\eta$ - installation efficiency ratio; $t$ - exposure to the energy.

Further simplifications are related to the exclusion of accounting for the electrochemical interaction of minerals with the environment and among themselves. By substitution, simplification, integration and counting $\eta, I, \sigma, B$ and $k$ permanent, addiction

$$
S_{n}=S_{0} \exp \{k \cdot B \cdot I[1-\operatorname{Aexp}(-2 \beta \cdot x)] \eta \cdot t / \sigma\},
$$

where $S_{0}$ - area of the interphase surface of particles at the beginning of the process (when $t=0)$.

$$
\text { Because } S=S_{y \partial} V \text {, то } S_{y \partial .0}=S_{0} / V \text {, where is } S_{y \partial} \text { - particle interphase surface; }
$$

$V$ - particle volume; $S_{y \partial .0}$ - particle interphasic surface when $t=0$, the addiction is converted to mind [1]:

$$
S_{y \partial}=S_{y \partial .0} \exp \left\{k \cdot B \cdot \sigma^{-1} \cdot I[1-A \exp (-2 \beta \cdot x)] \eta \cdot t\right\} .
$$

Developed a mathematical model of the process of formation of small particles mineral component of hydrosmesi in the field of hydrodynamic impact, initiated by ultrasound, as well as cavitation caused by hydrodynamic effects initiated by the use of installations [6-13], shows that the specific interphase surface of particles is expressed by dependence on the intensity of the energy emitted, the specific surface energy of the particles, the wave resistance of the hydrosmesi, the dissipation loss of the - distances from the source of radiation or cavitation, energy absorption by the environment, circular frequency of sound wave or cavitation, heat capacity and thermal conductivity, shift viscosity, volume viscosity, as well as the design features of the installation related to the efficiency of its operation. 
Based on experimental research, ways and installations have been developed to disintegrate [10-17]. The method of cavitation-hydrodynamic microdisintegration of the highly glides polymineral component of hydrosmesi [10] includes the high-speed flow of the jet, the processing of hydro-mineral mixture in conditions of active hydrodynamic influences by the influence of profiled tapering and expanding channels forming the hull of the hydrodynamic generator, as well as - consistently installed stationary cavitation elements in the form of beam-nooks, separation with the provision of deep disintegration of the polymineral component of hydro-mineral mixture to micro-level by converting the kinetic energy of the flow of liquid into the energy of acoustic vibrations. To amplify the fields of acoustic vibrations at the exit of the upper diffuser create turbulence, interdiction by a rigidly fixed top embarrassment and a cross mounted on the shelf, placed at the exit of the upper embarrassment, the upper beam-corners, the edges of the direct angle of which are turned up to the meeting by the stream, and installed vertically with a gap in relation to the wall of the upper embarrassment and a slope to the central part with an axis forming in the course of the hydro-mineral mixture movement an additional zone of narrowing. Created enrichment complex [18], contains a drop-off camera, vibrating device. The complex is equipped with pre-ultrasonic treatment, electromagnetic separation and demagnetization, as well as sensors for determining the degree of variance and the amplitude regulator of pulsations.

\section{Conclusion}

The identified basic dependency options allowed, experimental studies of the disintegration of mineral particles, using electron microscopy to control the process and assess its effectiveness. Developed ideas about the structural and mechanical features of hydro-mineral mixture, taking into account thermodynamic potential, create the prerequisites not only the theoretical justification of the process, but also the development of applied research in the development of technologies and means of processing and concentration of minerals in environmentally friendly ways.

It should be noted, that the prospect of research related to systems initiating various hydrodynamic effects of destruction, modeled by ultrasonic, electro-discharge, electromagnetic (Microwave), vibration and other hydrodynamic influences certainly require its further development.

\section{Literature}

1. N. P. Khrunina, O.V. Stratechuk, New aspects of the scientific and technological foundations of directional change of state and physical-mechanical properties of sand-clay rocks of gold-containing deposits (2007)

2. N. P. Khrunina, Yu A. Mamaev, A. M. Pulyaevsky, O.V. Stratechuk, New aspects of the scientific basis of ultrasonic disintegration of the high-glystic gold sands of the Priamurya scatterings (2011)

3. N. P. Khrunina, A. Yu. Cheban, Herald of Magnitogorsk State Technical University. G.I. Nosova. 4, 50 (2015)

4. Yu. A. Mamayev, N.P. Khrunina, Izvestia of higher education institutions. Mountain magazine. 6, 71 (2008)

5. Yu A. Mamayev, N.P. Khrunina, GIAB. S5, 47 (2009)

6. Yu A. Mamayev, N.P. Khrunina, GIAB. 7, 187 (2009)

7. N. P. Khrunina, A. Yu. Cheban. Georesources, 20, 1, 51 (2018)

8. N. P. Khrunina, Mountain Journal. 10, 39 (2018) 
9. N. P. Khrunina, Pat. 2200629 RU (2003)

10. N. P. Khrunina, Pat. 2714172 RU (2020)

11. N. P. Khrunina, Pat. 2714417 RU (2020)

12. N. P. Khrunina, Pat. 2117156 RU (1998)

13. N. P. Khrunina, Pat. 2187373 RU (2002)

14. N. P. Khrunina, Pat. 2209678 RU (2003)

15. N. P. Khrunina, Pat. 2206403 RU (2002)

16. N. P. Khrunina, Pat. 2204441 RU (2001)

17. N. P. Khrunina, Pat. 2209974 RU (2002)

18. N. P. Khrunina, Pat. 2231392 RU (2004) 